\title{
The predictive value of microRNAs for pathological response after neoadjuvant treatment in esophageal squamous cell carcinoma: a systematic review
}

\author{
Dong Lin, Xiaosang Chen, Lijie Tan \\ Department of Thoracic Surgery, Zhongshan Hospital of Fudan University, Shanghai, China \\ Contributions: (I) Conception and design: L Tan; (II) Administrative support: L Tan; (III) Provision of study materials or patients: D Lin, X Chen; (IV) \\ Collection and assembly of data: D Lin, X Chen; (V) Data analysis and interpretation: All authors; (VI) Manuscript writing: All authors; (VII) Final \\ approval of manuscript: All authors. \\ Correspondence to: Lijie Tan, MD, FACS. Department of Thoracic Surgery, Zhongshan Hospital of Fudan University, 180 Fenglin Road, Shanghai \\ 200032, China. Email: tan.lijie@zs-hospital.sh.cn.
}

\begin{abstract}
Neoadjuvant treatment followed by esophagectomy has been the standard strategy for resectable locally advanced esophageal squamous cell carcinoma (ESCC). Pathological response after neoadjuvant treatment is of vital importance in the determination of long-term survival. Due to the involvement of microRNAs (miRNAs) in ESCC, some studies have proposed miRNA models to predict the pathological response. We aimed to summarize current studies on the predictive value of the miRNA models. We searched the relevant studies on PubMed, Web of Science and Cochrane Library up to February 14, 2020, using the following search term: (esophageal OR esophagus OR oesophageal OR oesophagus) AND (miR OR miRNA OR microRNA) AND (neoadjuvant OR preoperative OR induction). The initial search retrieved 206 studies. We briefly summarized the involvement of miRNAs in the origin, development and chemo- and radioresistance in ESCC. Then, 9 studies were enrolled in the systematic review. A great heterogeneity was observed across these studies. Of the 6 studies with diagnostic tests, the area under curve varied a lot. Although much evidence demonstrated the correlation between miRNAs and pathological response after in ESCC, the current studies has not established any promising models. A well-designed prospective study is essential to investigate the potential predictive models for pathological response after neoadjuvant treatment in ESCC.
\end{abstract}

Keywords: Esophageal squamous cell carcinoma (ESCC); microRNAs (miRNA); pathological response; predictive

Submitted Apr 01, 2020. Accepted for publication Oct 23, 2020.

doi: 10.21037/atm-20-3000

View this article at: http://dx.doi.org/10.21037/atm-20-3000

\section{Introduction}

Esophageal cancer is one of the most common malignancies worldwide, which led to approximately 500,000 deaths in 2018 (1). Its two main histological subtypes, esophageal squamous cell carcinoma (ESCC) and esophageal adenocarcinoma (EAC), have significant differences in epidemiology, etiology, and treatment response (2). In East Asia and Africa, ESCC is the predominant subtype (3).

Patients with ESCC have poor overall survival, which is partly due to advanced stages at initial diagnosis (4), and the limited approaches for metastatic diseases $(5,6)$. In recent years, neoadjuvant treatment, including neoadjuvant chemotherapy (nCT) or neoadjuvant chemoradiotherapy (nCRT), followed by esophagectomy, has been the standard strategy for resectable locally advanced esophageal cancer due to the survival benefit (7-9). Moreover, the pathological response after nCT or nCRT has been demonstrated to be independently associated with overall survival $(10,11)$. Therefore, predicting pathological response is important. To date, researchers have proposed models based on 
medical images (12), inflammatory markers (13), and nutrient indices (14); however, no promising models have been established.

MicroRNAs (miRNAs), short nucleotide as $28-25$ base pairs, are considered potential candidates for predicting pathological response. They post-transcriptionally regulate gene expression, including that of oncogenes and onco-suppressor genes, by complementary binding to 3'-untranslated regions of the target messenger RNA. Previously published studies have reported that miRNAs are involved in the origin and development, and the chemoand radio-resistance of ESCC (15-20). In recent years, some studies have further explored whether miRNAs could effectively predict tumor response after nCT or nCRT in ESCC. However, variable miRNAs, together with different pathological response classifications, lead to complex outcomes that require further consideration.

In the present study, we briefly reviewed the involvement of miRNAs in the development and treatment resistance of ESCC. This systematic review aimed to analyze the predictive value of miRNAs in the pathological response of ESCC after nCT or nCRT. We present the following article in accordance with the PRISMA reporting checklist (available at http://dx.doi.org/10.21037/atm-20-3000) (21).

\section{Methods}

\section{Search strategy}

A literature search of PubMed, Web of Science, and Cochrane Library was conducted using the following search terms: (esophageal OR esophagus OR oesophageal OR oesophagus) AND (miR OR miRNA OR microRNA) AND (neoadjuvant OR preoperative OR induction). The search was restricted to English-language literature published from inception to February 14, 2020.

\section{Inclusion and exclusion criteria}

The inclusion criteria were as follows: (I) patients histologically diagnosed with esophageal cancer; (II) patients receiving $\mathrm{nCT}$ or nCRT followed by esophagectomy; and (III) studies on the association between miRNAs and pathological response to neoadjuvant treatment. The exclusion criteria were as follows: (I) published studies that were not original articles; (II) studies not on ESCC or miRNAs; and (III) studies not on pathological response. The reference lists of the original articles and literature reviews were also examined.

\section{Data extraction}

Data including first author, year of publication, sample size, neoadjuvant treatment, testing materials, pathological response classification, miRNA model, and statistical methods were extracted.

All procedures were independently double-checked by two investigators (D Lin and X Chen). Discrepancies were resolved through group discussions with all the authors.

\section{Statistical analysis}

It is well known that the part of Statistical Analysis is essential in original articles. However, this is a systematic review with no meta-analysis due to the heterogeneity across the enrolled studies. So, we think that the part of statistical analysis can be waived in our review article. We look forward to further communication at any time.

\section{Results}

\section{Study characteristics}

The initial search retrieved 206 studies. According to the inclusion and exclusion criteria, 197 studies were eliminated and 9 were finally selected for the review. The literature retrieval process is shown in Figure 1.

All 9 studies were published in the past decade (22-30). As summarized in Table 1, the 4 studies from Japan used nCT, while the other 5 studies, from China, Germany, and Canada, used nCRT. All chemotherapy regimens were platinum-based. The radiotherapy doses ranged from 30 to 60 Gy. Significant diversity was also observed in the testing materials, pathological response classifications, and the miRNA models. These findings indicated great heterogeneity among the studies, which eliminated the need for a quantitative analysis. The pathological response criteria are presented in Table S1.

\section{Involvement of miRNAs in the origin and development of ESCC}

In 2008, Guo et al. first reported the global microRNA expression profile of ESCC (31). They identified 46 miRNAs that were differentially expressed in malignant and adjacent normal tissue, of which 7 could distinguish between the 


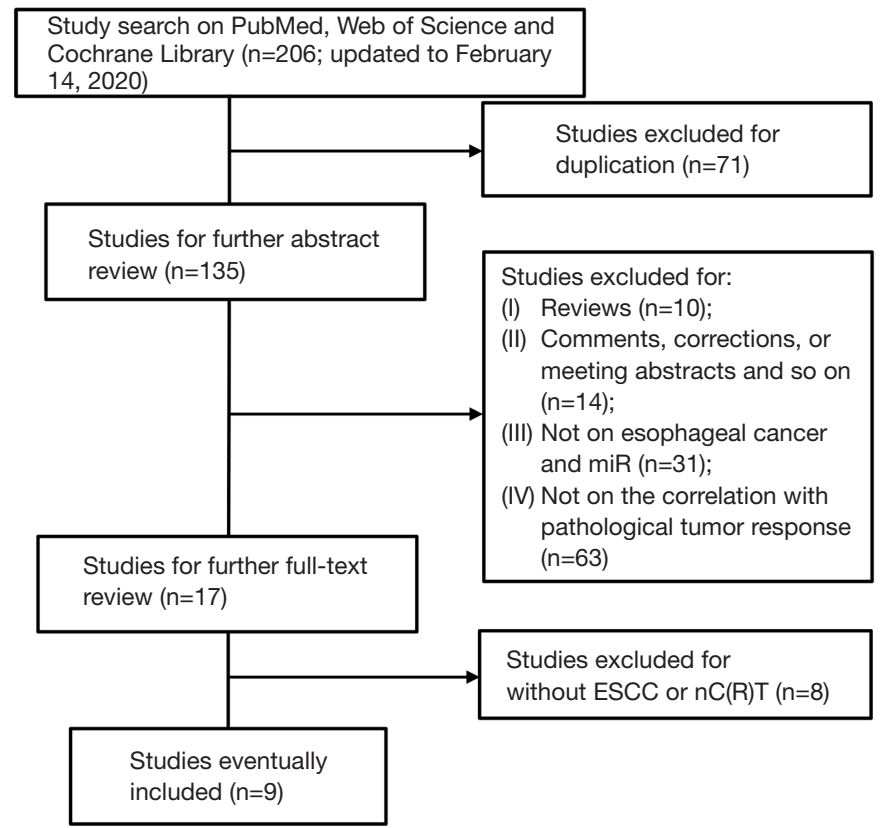

Figure 1 Flow diagram of study search and selection.

malignant and normal tissues. Moreover, they determined that miR-103/107 could be an independent prognostic marker. Kano et al. reported that the downregulation of miR-145, miR-133a, and miR-133b could lead to the activation of FSCN1, which promotes cell growth and invasion (32). Hamano et al. reported that miR-200c expression knockdown is associated with the increased expression of PPP2R1B, which might inhibit tumor invasiveness. Liu et al. revealed that ADAM9 is a key target of miR-126, and that the ectopic expression of miR126 or silencing of ADAM9 reduces the proliferation and migration abilities of ESCC cells (22). Harada et al. stated that the complicated dysregulation of miRNAs could be responsible for the origin and development of ESCC (19).

\section{Involvement of miRNAs in treatment sensitivity or resistance of ESCC}

Chen et al. revealed that the combined downregulation of miR-133a and miR-133b enhances chemosensitivity to paclitaxel-based chemotherapy (33), and miR-141 was revealed to confer resistance to cisplatin in ESCC (34). Zang et al. revealed that miR-199a-3p regulates radioresistance by targeting the AK4 gene (35). Moreover, miR96 promotes chemo- and radio-resistance in ESCC through the downregulation of RECK (36). Accumulating evidence has confirmed the associations between miRNAs and chemo- or radio-resistance, or chemo- or radio-sensitivity in ESCC. Vrana et al. stated that the role of miRNAs was interactive and complicated (20).

\section{Correlation between miRNAs and pathological response}

As mentioned above, the involvement of miRNAs in ESCC is an important precondition of potential predictive models. Recent studies have investigated whether single or panel miRNAs could effectively predict pathological response after nCT or nCRT in ESCC (25-30). As shown in Table 2, regarding the statistical methodology, 3 studies published from 2011-2013 that did not use diagnostic tests reported a correlation between miRNAs and pathological response. The other 6 studies, which were all published after 2013, used diagnostic tests, despite the absence of the STARD guidelines and external validation.

\section{Studies that did not use diagnostic tests}

In 2011, Hamano et al. reported that miR-200c was significantly correlated with pathological response $(\mathrm{P}=0.007)$ to nCT based on cisplatin, adriamycin and 5-FU (22). 
Table 1 Summary of nine studies on the miRNAs and pathological response after neoadjuvant treatment in ESCC

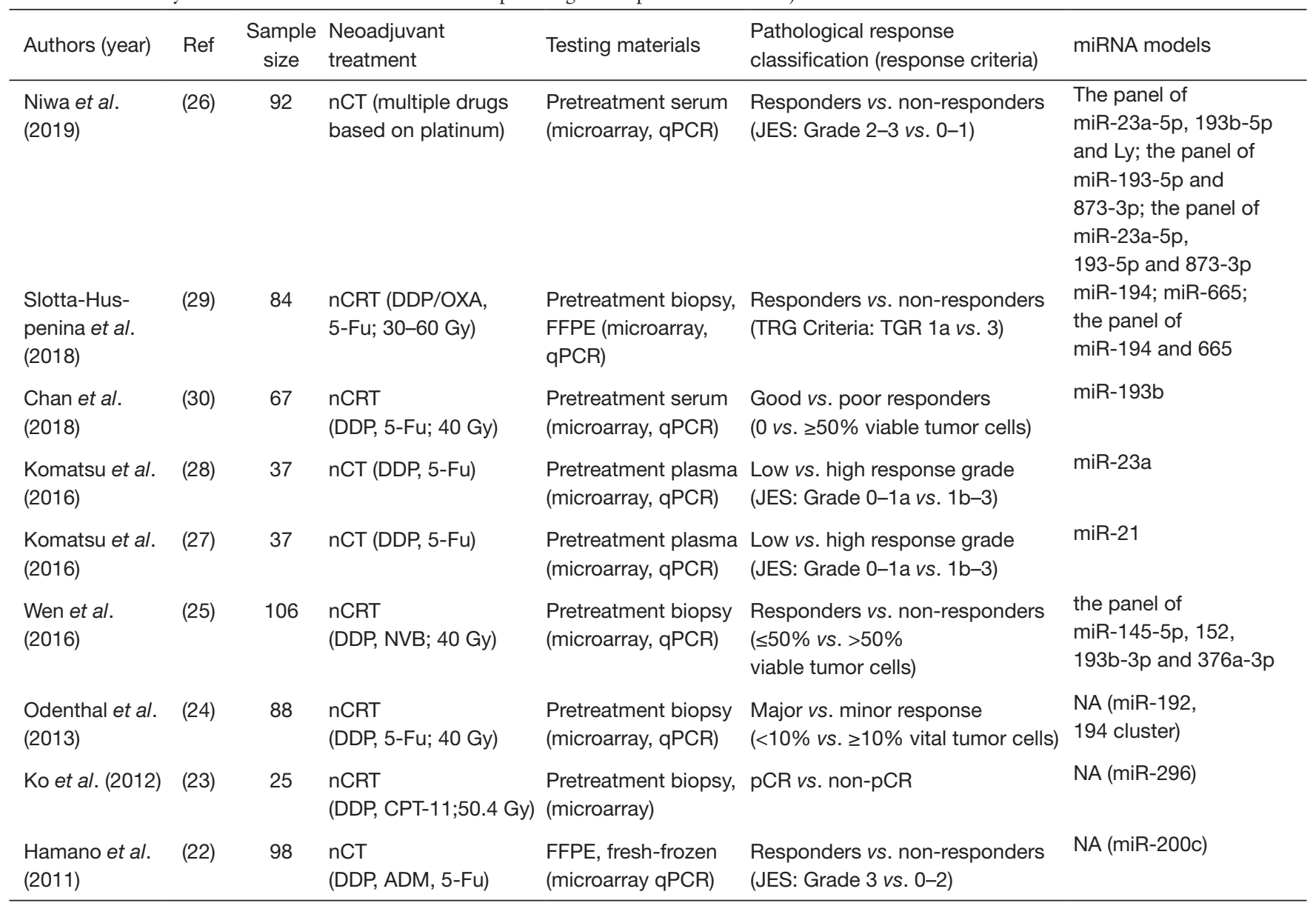

Ref, reference; Ly, lymphatic invasion; DDP, cisplatin; 5-Fu, fluorouracil; NVB, vinorelbine; CPT-11, irinotecan; ADM, adriamycin; JES, Japan Esophageal Society; TRG, tumor regression grade.

In a study of pathological complete response (pCR) and non-pCR patients, Ko et al. found that 71 miRNAs were significantly different, and 5 miRNAs had greater than 2 -fold differences (23). This study included 5 cases with ESCC and 20 with EAC. Odenthal et al. reported that the expression of miR-192 ( $\mathrm{P}=0.005)$ and miR-194 $(\mathrm{P}=0.040)$ were significantly different between ESCC patients who showed minor and major response (24). Further, these studies found that the expression levels of specific miRNAs changed significantly before and after nCT or nCRT. These dynamic changes offered a possibility of early evaluation during neoadjuvant treatment. These studies revealed a significant correlation between miRNAs and pathological response.

\section{Studies that used diagnostic tests}

In 2016, Wen et al. identified 10 miRNAs with greater than 1.5-fold changes between pathological responders $(<50 \%$ residual tumor) and non-responders $(\geq 50 \%$ residual tumor), and established a combination of 4 miRNAs (miR145-5p, miR-152, miR-193-3p, and miR-376a-3p) as a predictive model (25). The model provided a satisfactory predictive value for pathological response in an external validation cohort [area under the curve (AUC): 0.8682, 95\% confidence interval $(\mathrm{CI}): 0.7710-0.9653, P<0.001]$. In this study, patients underwent homogeneous concurrent nCRT. But the underlying mechanism of these miRNAs was still unclear. 
Table 2 Summary of six studies with diagnostic tests on the predictive value of miRNA for pathological response after neoadjuvant treatment in ESCC

\begin{tabular}{|c|c|c|c|c|c|c|}
\hline Authors (year) & Ref & $\begin{array}{c}\text { Sample size } \\
\text { of external } \\
\text { validation }\end{array}$ & Methods of diagnostic tests & $\begin{array}{l}\text { Most valuable } \\
\text { predictive models }\end{array}$ & \multicolumn{2}{|c|}{ Predictive power (at the optimal cutoff) } \\
\hline $\begin{array}{l}\text { Slotta-Huspen- } \\
\text { ina et al. (2018) }\end{array}$ & $(29)$ & NA & $\mathrm{ROC}$ analysis & $\begin{array}{l}\text { the panel of miR-194 and } \\
\text { miR-665 }\end{array}$ & $\begin{array}{c}0.824 \\
(0.713-0.935)\end{array}$ & No data \\
\hline $\begin{array}{l}\text { Komatsu et al. } \\
\text { (2016) }\end{array}$ & $(28)$ & NA & ROC analysis & miR-23a & 0.696 (no data) & $\begin{array}{l}\text { Sen: } 79.2 \% \text {, } \\
\text { Spe: } 64.3 \%\end{array}$ \\
\hline $\begin{array}{l}\text { Komatsu et al. } \\
(2016)\end{array}$ & $(27)$ & NA & $\begin{array}{l}\text { ROC analysis with Youden } \\
\text { index }\end{array}$ & $\mathrm{miR}-21$ & 0.6794 (no data) & $\begin{array}{l}\text { Sen: } 92.3 \% \text {, Spe: } \\
54.2 \% \text {, Acc: } 67.6 \%\end{array}$ \\
\hline
\end{tabular}

Ref, reference; ROC, receiver operative characteristic curve; AUC, area under the curve; 95\% Cl, 95\% confidence interval; Ly, lymphatic invasion; JES, Japan Esophageal Society; TRG, tumor regression grade; Sen, sensitivity; Spe, specificity; Acc, accuracy.

In recent years, circulating miRNAs have attracted significant attention due to their non-invasiveness and convenience. In 2019, Niwa et al. conducted serumbased miRNA signature research, which was carried out in accordance with the STARD guidelines (26). They identified 62 miRNAs in responders (grades 2-3) and non-responders (grade 0-1) after nCT. MiR-193b-5p, miR-873-3p, and miR-23a-5p, as well as microscopic lymphatic invasion, were included in the models. Consequently, receiver-operative characteristic (ROC) analysis confirmed that the combination of miR-193b$5 \mathrm{p}, \mathrm{miR}-873-3 \mathrm{p}$, and lymphatic invasion achieved the highest predictive value (AUC: $0.73,95 \%$ CI: 0.60-0.86; no sensitivity, specificity, or accuracy data). However, the models did not show any independent prognostic value for long-term survival.

Komatsu et al. proposed miR-21 (27) and miR-23a (28) from pretreatment plasm as useful biomarkers for pathological response. However, these 2 miRNAs were found to have only low predictive value (AUC: 0.6794, sensitivity: $92.3 \%$, specificity: $54.2 \%$, and accuracy: $67.6 \%$; AUC: 0.696 , sensitivity: $79.2 \%$, and specificity: $64.2 \%$, respectively).

Slotta-Huspenina et al. identified 12 miRNAs from 15 responders [tumor regression grade (TRG) $1 \mathrm{a}$ ] and 16 non-responders (TRG 3) after nCRT (29). ROC analysis confirmed the predictive value of miR$194^{*}$, miR-665, and their combination for pathological response (AUC: 0.811, 0.817, and 0.824, respectively) in the expanded 53 cases including 26 responders (TRG 1a) and 27 non-responders (TRG 3) after nCRT. Similarly, Chan et al. identified 3 miRNAs from 10 good responders (with no viable tumor cells) and 10 poor responders ( $\geq 50 \%$ viable tumor cells) after nCRT (30). The subsequent validation among 24 patients with good response (with no viable tumor cells) and 23 patients with poor response ( $\geq 50 \%$ viable tumor cells) revealed that miR$193 \mathrm{~b}$ had a strong predictive power to discriminate between the patients (AUC: 0.8949, 95\% CI: 0.7912-0.9987, $P<0.0001)$.

\section{Discussion}

Pathological response is of importance for long-term survival after neoadjuvant treatment $(10,11)$. Therefore, numerous efforts have been made to establish predictive models. In the past decade, miRNAs have been the focus of attention in cancer research. Vrana et al. analyzed the involvement of miRNAs in the origin and development of ESCC, and in the chemo- and radio-resistance of 
ESCC (20). All of these formed one precondition for the hypothesis that miRNAs might be biomarkers for pathological response.

The aim of our review was to analyze studies on the predictive value of miRNAs for pathological response after nCT or nCRT in ESCC. After the selection and evaluation of articles, however, we observed great heterogeneity among the studies, and their methodologies and outcomes required further consideration.

Of the 6 studies that used diagnostic tests, 3 reported AUCs $>0.80$. Wen $e t a l$. proposed a predictive panel of miRNAs miR-145-5p, 152, 193b-3p, and 376a-3p in patients undergoing homogeneous nCRT (25). The effects of these miRNAs on tumor invasion varied. MiR$145-5 p$ has been found to have an oncogenic function in esophageal cancer (37), while the overexpression of miR152 sensitizes cisplatin-resistant ovarian cancer cells (38). Meanwhile, miR-193b-3p and 376a-3p have been shown to be tumor suppressors $(39,40)$. In this study, however, Wen et al. did not investigate its prognostic value. In our opinion, complete or major pathological response is an indicator of long-term survival. Survival benefit, rather than pathological response, is the aim of any potential predictive model. An ideal model with the aims of predicting response and selecting patients should also have an independent association with survival benefit.

Apart from Wen et al.'s study, Slotta-Huspenina et al. (29) and Chan et al. (30) respectively reported models with high predictive values. However, in these latter 2 studies, we observed that the miRNA candidates were identified from 2 extreme pathological response classifications: TRG1a and TRG3 in Slotta-Huspenina et al.'s study, and no viable tumor cells versus $\geq 50 \%$ viable tumor cells in Chan et al.'s study. The spectrum bias would overestimate the predictive power (41). Moreover, the models were validated among patients with extreme pathological response classifications. The models offer limited value for decision-making in the clinical setting. Therefore, future well-designed studies that adhere to the standard guidelines of diagnostic tests are essential (42).

All 9 studies used pretreatment specimens as the testing materials. The studies in the early period had found the dynamics of miRNAs before and after nCT or nCRT. The possibility of early evaluation during neoadjuvant treatment could be an important direction. Four studies used serum or plasma specimens as the testing materials. Circulating miRNAs could serve as sensitive and informative biomarkers for certain diseases (43). However, inconsistencies between miRNAs in serum and miRNAs in tumor tissues have been reported (44). The use of miRNA panels, with or without clinicopathological factors, was also observed to have increased in recent years. The combination of miRNAs and other potential biomarkers, such as medical images, inflammatory markers, and nutrient indices, deserves further investigation.

Our systematic review has several limitations. First, the retrospective nature of the enrolled studies led to inherent selection bias. Second, only studies published in English were enrolled, leading to publication bias. Third, heterogeneity existed in treatment regimens, response classifications, and miRNA panels across the enrolled studies. Although previous studies have comprehensively analyzed the involvement of miRNAs in ESCC treatment resistance (20), the present review concentrated on the predictive value of miRNAs in pathological response after nCT or nCRT in ESCC. Despite the limited number of enrolled studies, our review uncovered some issues and proposed our opinions, and these would be helpful to further studies.

\section{Conclusions}

More and more evidence has demonstrated the correlation between miRNAs and pathological response after $\mathrm{nCT}$ and nCRT in ESCC. However, the current studies had not established any promising model. A well-designed prospective study is essential to investigate the potential models for pathological response after neoadjuvant treatment in ESCC.

\section{Acknowledgments}

We thank Dr. Jiaying Deng (Fudan University Shanghai Cancer Center) for his contribution to the search strategy. We thank Xuejuan Jin (Zhongshan Hospital of Fudan University) for her contribution to the statistical interpretation in this article. We acknowledge R. Scott and J. Reynolds from AME Editing Service for their help in language editing.

Funding: This study was supported by the Fund of Zhongshan Hospital of Fudan University (No. 2019ZSFZ16, No. 2016ZSLC15). The funding had no impact on study design, data collection, data interpretation or manuscript writing. 


\section{Footnote}

Reporting Checklist: The authors have completed the PRISMA reporting checklist. Available at http://dx.doi. org/10.21037/atm-20-3000

Conflicts of Interest: All authors have completed the ICMJE uniform disclosure form (available at http://dx.doi. org/10.21037/atm-20-3000). The authors have no conflicts of interest to declare.

Ethical Statement: The authors are accountable for all aspects of the work in ensuring that questions related to the accuracy or integrity of any part of the work are appropriately investigated and resolved. The data used in this study are from the database, so ethical approval and informed consent of the patient are not required.

Open Access Statement: This is an Open Access article distributed in accordance with the Creative Commons Attribution-NonCommercial-NoDerivs 4.0 International License (CC BY-NC-ND 4.0), which permits the noncommercial replication and distribution of the article with the strict proviso that no changes or edits are made and the original work is properly cited (including links to both the formal publication through the relevant DOI and the license). See: https://creativecommons.org/licenses/by-nc-nd/4.0/.

\section{References}

1. Bray F, Ferlay J, Soerjomataram I, et al. Global cancer statistics 2018: GLOBOCAN estimates of incidence and mortality worldwide for 36 cancers in 185 countries. CA Cancer J Clin 2018;68:394-424.

2. Rustgi AK, El-Serag HB. Esophageal carcinoma. N Engl J Med 2014;371:2499-509.

3. Abnet CC, Arnold M, Wei WQ. Epidemiology of Esophageal Squamous Cell Carcinoma. Gastroenterology 2018;154:360-73.

4. Herskovic A, Russell W, Liptay M, et al. Esophageal carcinoma advances in treatment results for locally advanced disease: review. Ann Oncol 2012;23:1095-103.

5. Kashyap MK, Abdel-Rahman O. Expression, regulation and targeting of receptor tyrosine kinases in esophageal squamous cell carcinoma. Mol Cancer 2018;17:54.

6. Kono K, Mimura K, Yamada R, et al. Current status of cancer immunotherapy for esophageal squamous cell carcinoma. Esophagus 2018;15:1-9.

7. Lordick F, Mariette C, Haustermans K, et al. Oesophageal cancer: ESMO Clinical Practice Guidelines for diagnosis, treatment and follow-up. Ann Oncol 2016;27:v50-7.

8. Kuwano H, Nishimura Y, Oyama T, et al. Guidelines for Diagnosis and Treatment of Carcinoma of the Esophagus April 2012 edited by the Japan Esophageal Society. Esophagus 2015;12:1-30.

9. Ajani JA, D'Amico TA, Bentrem DJ, et al. Esophageal and Esophagogastric Junction Cancers, Version 2.2019, NCCN Clinical Practice Guidelines in Oncology. J Natl Compr Canc Netw 2019;17:855-83.

10. Meredith KL, Weber JM, Turaga KK, et al. Pathologic response after neoadjuvant therapy is the major determinant of survival in patients with esophageal cancer. Ann Surg Oncol 2010;17:1159-67.

11. Tomasello G, Petrelli F, Ghidini M, et al. Tumor regression grade and survival after neoadjuvant treatment in gastro-esophageal cancer: A meta-analysis of 17 published studies. Eur J Surg Oncol 2017;43:1607-16.

12. Lordick F, Ott K, Krause BJ, et al. PET to assess early metabolic response and to guide treatment of adenocarcinoma of the oesophagogastric junction: the MUNICON phase II trial. Lancet Oncol 2007;8:797-805.

13. Fang P, Jiang W, Davuluri R, et al. High lymphocyte count during neoadjuvant chemoradiotherapy is associated with improved pathologic complete response in esophageal cancer. Radiother Oncol 2018;128:584-90.

14. Ota T, Ishikawa T, Endo Y, et al. Skeletal muscle mass as a predictor of the response to neo-adjuvant chemotherapy in locally advanced esophageal cancer. Med Oncol 2019;36:15.

15. Sakai NS, Samia-Aly E, Barbera M, et al. A review of the current understanding and clinical utility of miRNAs in esophageal cancer. Semin Cancer Biol 2013;23:512-21.

16. Gusella M, Pezzolo E, Modena Y, et al. Predictive genetic markers in neoadjuvant chemoradiotherapy for locally advanced esophageal cancer: a long way to go. Review of the literature. Pharmacogenomics J 2018;18:14-22.

17. Li Y, Huang HC, Chen LQ, et al. Predictive biomarkers for response of esophageal cancer to chemo(radio)therapy: A systematic review and meta-analysis. Surg Oncol 2017;26:460-72.

18. Mei LL, Qiu YT, Zhang B, et al. MicroRNAs in esophageal squamous cell carcinoma: Potential biomarkers and therapeutic targets. Cancer Biomark 2017;19:1-9.

19. Harada K, Baba Y, Ishimoto T, et al. The role of 
microRNA in esophageal squamous cell carcinoma. J

Gastroenterol 2016;51:520-30.

20. Vrana D, Matzenauer M, Aujesky R, et al. Potential Predictive Role of MicroRNAs in the Neoadjuvant Treatment of Esophageal Cancer. Anticancer Res 2017;37:403-12.

21. Moher D, Liberati A, Tetzlaff J, et al. Preferred reporting items for systematic reviews and meta-analyses: the PRISMA statement. J Clin Epidemiol 2009;62:1006-12.

22. Hamano R, Miyata H, Yamasaki M, et al. Overexpression of miR-200c induces chemoresistance in esophageal cancers mediated through activation of the Akt signaling pathway. Clin Cancer Res 2011;17:3029-38.

23. Ko MA, Zehong G, Virtanen C, et al. MicroRNA expression profiling of esophageal cancer before and after induction chemoradiotherapy. Ann Thorac Surg 2012;94:1094-102; discussion 102-3.

24. Odenthal M, Bollschweiler E, Grimminger PP, et al. MicroRNA profiling in locally advanced esophageal cancer indicates a high potential of miR-192 in prediction of multimodality therapy response. Int J Cancer 2013;133:2454-63.

25. Wen J, Luo K, Liu H, et al. MiRNA Expression Analysis of Pretreatment Biopsies Predicts the Pathological Response of Esophageal Squamous Cell Carcinomas to Neoadjuvant Chemoradiotherapy. Ann Surg 2016;263:942-8.

26. Niwa Y, Yamada S, Sonohara F, et al. Identification of a serum-based miRNA signature for response of esophageal squamous cell carcinoma to neoadjuvant chemotherapy. J Transl Med 2019;17:1.

27. Komatsu S, Ichikawa D, Kawaguchi T, et al. Circulating miR-21 as an independent predictive biomarker for chemoresistance in esophageal squamous cell carcinoma. Am J Cancer Res 2016;6:1511-23.

28. Komatsu S, Ichikawa D, Kawaguchi T, et al. Plasma microRNA profiles: identification of miR-23a as a novel biomarker for chemoresistance in esophageal squamous cell carcinoma. Oncotarget 2016;7:62034-48.

29. Slotta-Huspenina J, Drecoll E, Feith M, et al. MicroRNA expression profiling for the prediction of resistance to neoadjuvant radiochemotherapy in squamous cell carcinoma of the esophagus. J Transl Med 2018;16:109.

30. Chan CM, Lai KKY, Ng EKO, et al. Serum microRNA$193 \mathrm{~b}$ as a promising biomarker for prediction of chemoradiation sensitivity in esophageal squamous cell carcinoma patients. Oncol Lett 2018;15:3273-80.

31. Guo Y, Chen Z, Zhang L, et al. Distinctive microRNA profiles relating to patient survival in esophageal squamous cell carcinoma. Cancer Res 2008;68:26-33.

32. Kano M, Seki N, Kikkawa N, et al. miR-145, miR-133a and miR-133b: Tumor-suppressive miRNAs target FSCN1 in esophageal squamous cell carcinoma. Int J Cancer 2010;127:2804-14.

33. Chen G, Peng J, Zhu W, et al. Combined downregulation of microRNA-133a and microRNA-133b predicts chemosensitivity of patients with esophageal squamous cell carcinoma undergoing paclitaxel-based chemotherapy. Med Oncol 2014;31:263.

34. Imanaka Y, Tsuchiya S, Sato F, et al. MicroRNA-141 confers resistance to cisplatin-induced apoptosis by targeting YAP1 in human esophageal squamous cell carcinoma. J Hum Genet 2011;56:270-6.

35. Zang C, Zhao F, Hua L, et al. The miR-199a-3p regulates the radioresistance of esophageal cancer cells via targeting the AK4 gene. Cancer Cell Int 2018;18:186.

36. Xia H, Chen S, Chen K, et al. MiR-96 promotes proliferation and chemo- or radioresistance by downregulating RECK in esophageal cancer. Biomed Pharmacother 2014;68:951-8.

37. Akagi I, Miyashita M, Ishibashi O, et al. Relationship between altered expression levels of MIR21, MIR143, MIR145, and MIR205 and clinicopathologic features of esophageal squamous cell carcinoma. Dis Esophagus 2011;24:523-30.

38. He J, Yu JJ, Xu Q, et al. Downregulation of ATG14 by EGR1-MIR152 sensitizes ovarian cancer cells to cisplatininduced apoptosis by inhibiting cyto-protective autophagy. Autophagy 2015;11:373-84.

39. Li J, Kong F, Wu K, et al. miR-193b directly targets STMN1 and uPA genes and suppresses tumor growth and metastasis in pancreatic cancer. Mol Med Rep 2014;10:2613-20.

40. Zheng Y, Yin L, Chen H, et al. miR-376a suppresses proliferation and induces apoptosis in hepatocellular carcinoma. FEBS Lett 2012;586:2396-403.

41. Lijmer JG, Mol BW, Heisterkamp S, et al. Empirical evidence of design-related bias in studies of diagnostic tests. JAMA 1999;282:1061-6.

42. Bossuyt PM, Reitsma JB, Bruns DE, et al. STARD 2015: an updated list of essential items for reporting diagnostic accuracy studies. BMJ 2015;351:h5527.

43. Wang K, Zhang S, Marzolf B, et al. Circulating microRNAs, potential biomarkers for drug-induced liver injury. Proc Natl Acad Sci U S A 2009;106:4402-7. 
44. Kurashige J, Kamohara H, Watanabe M, et al. Serum microRNA-2 1 is a novel biomarker in patients with

Cite this article as: Lin D, Chen X, Tan L. The predictive value of microRNAs for pathological response after neoadjuvant treatment in esophageal squamous cell carcinoma: a systematic review. Ann Transl Med 2021;9(5):420. doi: 10.21037/atm-203000 esophageal squamous cell carcinoma. J Surg Oncol 2012;106:188-92. 


\section{Supplementary}

Table S1 Summary of criteria of pathological evaluation in the selected studies

\begin{tabular}{|c|c|}
\hline \multirow{6}{*}{$\begin{array}{l}\text { Japan } \\
\text { Esophageal } \\
\text { Society } \\
\text { (JES criteria) }\end{array}$} & Grade 0: No recognizable cytological or histological therapeutic effect \\
\hline & $\begin{array}{l}\text { Grade1: Apparently viable cancer cells (including cells having eosinophilic cytoplasm with vacuolation and swollen nuclei) } \\
\text { account for } 1 / 3 \text { or more of tumor tissue, but there is some evidence of degeneration of cancer tissue or cells }\end{array}$ \\
\hline & 1a: Viable cancer cells accounting for $2 / 3$ or more tumor tissue \\
\hline & $1 \mathrm{~b}$ : Viable cancer cells accounting for $1 / 3$ or more, but less than $2 / 3$, of tumor tissue \\
\hline & $\begin{array}{l}\text { Grade 2: Viable cancer cells account for less than } 1 / 3 \text { of tumor tissue, while other cancer cells are severely degenerated or } \\
\text { necrotic }\end{array}$ \\
\hline & Grade 3: No viable cancer cells are evident \\
\hline \multirow{4}{*}{$\begin{array}{l}\text { Tumor } \\
\text { regression } \\
\text { grades } \\
\text { (TRG criteria) }\end{array}$} & TRG 1a: No residual tumor/tumor bed \\
\hline & TRG $1 \mathrm{~b}:<10 \%$ residual tumor/tumor bed \\
\hline & TRG 2: $10-50 \%$ residual tumor/tumor bed \\
\hline & TRG 3: $>50 \%$ residual tumor/tumor bed \\
\hline
\end{tabular}

